\title{
A note on enumeration of one-vertex maps ${ }^{*}$
}

\author{
Alen Orbanić, Marko Petkovšek \\ Tomaž Pisanski ${ }^{\dagger}$, Primož Potočnik \\ Institute of Mathematics, Physics and Mechanics and \\ Faculty of Mathematics and Physics, University of Ljubljana \\ Jadranska 19, SI-1000 Ljubljana, Slovenia
}

Received 29 December 2008, accepted 18 December 2009, published online 9 January 2010

\begin{abstract}
Explicit formulæ for the numbers of distinct maps and pre-maps having a single vertex of valence $d$ are given. The question of what precisely is meant by a map can be answered in several different ways. We enumerate 16 different types of objects where each type is obtained by selecting whether the object is a pre-map or a map, is oriented or general, whether the underlying graph or pre-graph is signed or unsigned, is directed or undirected.
\end{abstract}

Keywords: Oriented maps and pre-maps, orientable maps and pre-maps, non-orientable maps and pre-maps, pre-graphs, matchings in complete graphs, Cauchy-Frobenius-Burnside lemma.

Math. Subj. Class.: 05C10, 05C30

\section{Introduction}

It may seem that enumerating one-vertex maps looks rather sterile. On the other hand, a natural approach to vertex-transitive maps, such as Cayley maps (see Malnič, Nedela and Škoviera [17] and Richter, Širáň, Jajcay, Tucker and Watkins [21]), is to take the quotient by the automorphism group, or a transitive subgroup of the automorphism group, which automatically gives a one-vertex map. Thus any understanding of vertex-transitive maps depends on an understanding of one-vertex maps. Furthermore, taking quotients by group actions leads to a variety of "degenerate" structures, like semi-edges, which might not arise from an intuitive idea of a map and which have not been treated consistently and uniformly in the study of maps. The purpose of this paper is to provide a general setting for maps

\footnotetext{
* The authors acknowledge partial funding of this research by ARRS of Slovenia, grants: L2-7230, P1-0294, L2-7207 and the PASCAL network of excellence in the 6th framework.

$\dagger$ Joint position at the University of Primorska.

E-mail addresses: alen.orbanic@fmf.uni-lj.si (Alen Orbanić), marko.petkovsek@fmf.uni-lj.si (Marko Petkovšek), tomaz.pisanski@fmf.uni-lj.si (Tomaž Pisanski), primoz.potocnik@fmf.uni-lj.si (Primož Potočnik)
} 
allowing such degenerate structures from the outset, and then using that setting to analyse and enumerate one-vertex maps.

We should make it clear that the theory of combinatorial maps has a rich history. Unfortunately, there were several equivalent, but not identical, approaches to maps introduced in the past. In particular, treatment of oriented maps (on orientable surfaces) is much simpler than the treatment of general maps that may result in orientable or non-orientable surfaces. When considering maps as graphs, embedded in surfaces, the approach by Heffter [11], later formalized by Stahl [26], with the so-called generalized embedding scheme for general maps (orientable or not) is quite useful. The reader is referred to the three classical textbooks in topological graph theory; see [22, 8, 31]. Unfortunately, this approach was not developed for pre-maps, i.e., maps with underlying pre-graphs admitting semi-edges. An alternative way of presenting maps is via specifying three involutions [5].

Let us also point out that the field of enumeration of maps, even one-vertex maps, is very rich and well-established. Certain problems of enumerating isomorphism classes of one-vertrex maps were addressed by Gross, Robbins and Tucker in [7], by Cori and Marcus in [4], by Stoimenow in [28], by Khruzin in [13] ${ }^{1}$, by Kwak and Shim in [14], and by Chapuy in [2]. An approach using advanced techniques, such as moduli spaces, was suggested in a well-known work by Harer and Zagier [10], where they compute the number of one-vertex maps, not only according to the number of edges but according to the number of edges AND the number of faces (i.e., the genus of the surface). We would like to make it clear that maps in this paper are not being counted by genus; the formulæ give the total number of maps of orientable genus $g$ over all possible $g$. In the case of pre-maps, we also count the maps on surfaces with boundary. In the case of general maps, the enumeration also applies to the non-orientable genus.

Also, there are now several bijective interpretations of the enumerative formulæ found by Harer and Zagier, in particular by Goulden and Nica [6]. The reader is referred to the work of Liskovets such as [15] where he develops techniques similar to ours. Standard counting techniques to enumerate simple structures (like dissections of a polygon) up to symmetry can be found, for instance, in the book by Harary and Palmer [9]. Similarly, check the work of Walsh and Lehman [30] and Robinson [23].

In this article, we consider three distinct boolean parameters giving rise to eight enumeration problems:

First, we distinguish between maps and pre-maps. This parameter is needed since we are mostly interested in counting pre-maps, but topologically maps are more natural objects. Note that maps correspond to Cayley graphs with no involutory generators.

The second parameter is the choice of directed vs. undirected loops. In our setting this corresponds to the choice whether we consider Cayley graphs as directed or not, or equivalently, whether we select in each pair consisting of a generator and its inverse one element as the "preferred one".

Our final parameter is the possibility to give a sign to some loops. Such a sign defines a "twist" in the regular neighborhood of the corresponding non-involutory generator of the Cayley graph, thereby extending the definition of the Cayley map to non-orientable surfaces. Note that the actual Cayley map may still reside on an orientable surface (if the order of the generator is even, and some other conditions are met; see for instance [8].) The fact that the graph is signed or not is not a big problem in enumeration of one-vertex maps.

\footnotetext{
${ }^{1}$ Note that the value given in $\left[13\right.$, p. 8] for $d_{11}=\delta_{\bar{S} \bar{D} \bar{P}}(22)$ is incorrect.
} 
It just consists in giving one of two possible colors (say red or blue) to each loop. This makes it equivalent with enumeration of loop-bicolored (oriented!) maps.

We introduce a structure that we call a pre-graph; see also [17]. A pre-graph $G$ is a quadruple $G=(V, A, i, r)$ where $V$ is the set of vertices, $A$ is the set of arcs (also known as semi-edges, darts, sides, ...), $i$ is the initial mapping $i: A \rightarrow V$ specifying the origin or initial vertex for each arc, while $r$ is the reversal involution: $r: A \rightarrow A, r^{2}=1$. We may also define the terminal mapping $t: A \rightarrow V$ as $t(s):=i(r(s))$, specifying the terminal vertex for each arc. An arc $s$ forms an edge $e=\{s, r(s)\}$, which is called proper if $|e|=2$ and is called a half-edge if $|e|=1$. Define $\partial(e)=\{i(s), t(s)\}$. A pre-graph without half-edges is called a (general) graph. Note that $G$ is a graph if and only if the reversal involution has no fixed points. A proper edge $e$ with $|\partial(e)|=1$ is called a loop and two edges $e, e^{\prime}$ are parallel if $\partial(e)=\partial\left(e^{\prime}\right)$. A graph without loops and parallel edges is called simple. The valence of a vertex $v$ is defined as $\operatorname{val}(v)=|\{s \in A \mid i(s)=v\}|$. All pre-graphs in this note are connected unless stated otherwise.

Topologically, an oriented map is a 2-cell embedding of a graph into an oriented closed surface. However, in this paper we will operate with the following combinatorial description. For us an oriented pre-map is a triple $(A, r, R)$ where $A$ is a finite non-empty set of darts and $r, R$ are permutations on $A$ such that $r^{2}=1$ and $\langle r, R\rangle$ acts transitively on $A$ (see [17], but the description goes back to Jacques [12] and Cori [3]). Note that the vertices of the pre-map correspond to the cycles in the cyclic decomposition of $R$. An isomorphism between two pre-maps $(A, r, R)$ and $\left(A^{\prime}, r^{\prime}, R^{\prime}\right)$ is any bijection $\pi: A \rightarrow A^{\prime}$ for which $\pi R=R^{\prime} \pi$ and $\pi r=r^{\prime} \pi$ holds. An automorphism of a map $(A, r, R)$ is thus a permutation of $A$ which commutes with both $R$ and $r$. If $r$ is fixed-point free, the structure is called an oriented map rather than a pre-map. Combinatorial oriented maps correspond to topological maps on oriented closed surfaces while oriented pre-maps correspond to topological maps on surfaces with boundary (with a natural orbifold structure). To each oriented pre-map $M=(A, r, R)$ we may associate a unique map with reversed orientation $M^{-1}=\left(A, r, R^{-1}\right)$. Each oriented map gives rise to the automorphism group $\operatorname{Aut}_{0}(M)$ of all (orientation-preserving) automorphisms, which can be extended to a larger group $\operatorname{Aut}(M)$ by allowing the orientation-reversing automorphisms, i.e., isomorphisms from $(A, r, R)$ to $\left(A, r, R^{-1}\right)$. Note that the index of $\operatorname{Aut}_{0}(M)$ in $\operatorname{Aut}(M)$ is either 1 or 2 . If it is 1 then $M$ is chiral.

In addition to oriented maps we will also consider general (possibly non-orientable) (pre-)maps, which can be defined as $M=(A, r, R, \lambda)$, where $A, r$ and $R$ are as in the definition of oriented maps, and $\lambda$ is a sign mapping assigning either 1 or -1 to each proper edge of the underlying pre-graph of the map $M$. Recall that each cycle $C=\left(s_{1}, \ldots, s_{k}\right)$ of $R$ corresponds to a vertex of the map. Substituting the cycle $C$ in $R$ with its reverse cycle and inverting the $\lambda$-value of proper edges underlying the darts $s_{1}, \ldots, s_{k}$ other than loops, results in a new map $M^{\prime}$, which is said to be obtained from $M$ by a local orientation change. Two maps $M_{1}=\left(A_{1}, r_{1}, R_{1}, \lambda_{1}\right)$ and $M_{2}=\left(A_{2}, r_{2}, R_{2}, \lambda_{2}\right)$ are isomorphic if there exists a map $M^{\prime}=\left(A_{1}, r_{1}, R^{\prime}, \lambda^{\prime}\right)$ obtained from $M_{1}$ by a series of local orientation changes, and a bijection $\pi: A_{1} \rightarrow A_{2}$, such that $\pi r_{1}=r_{2} \pi, \pi R^{\prime}=R_{2} \pi$ and $\lambda^{\prime}=$ $\lambda_{2} \pi$. One of the early definitions of general maps goes back to Tutte [29], his definition is equivalent to ours.

Note that in the topological interpretation a general pre-map resides in a surface that may be orientable or non-orientable. There is a well-known condition for testing the orientability of this surface. Namely the underlying surface is non-orientable if and only if 
there is a cycle with the product of signs $\lambda$ along its edges equal to -1 .

There is a well-known relationship between oriented maps and maps that reside on orientable surfaces, namely each pair of oriented maps $(A, r, R)$ and $\left(A, r, R^{-1}\right)$ gives rise to the same map $(A, r, R, \lambda)$ where $\lambda$ is the constant 1 , while each orientable map admits a representation of such form and gives rise to two oriented maps: $(A, r, R)$ and $\left(A, r, R^{-1}\right)$.

In case of embeddings of directed graphs or pre-graphs we may speak, respectively, of directed maps or directed pre-maps.

We refer the reader to [20] for further details on the theory of maps and embeddings of graphs and pre-graphs into surfaces. For the theory of Cayley maps, see for instance [21]. An overview of a combinatorial approach to coverings of graphs and pre-graphs was given in [19].

The main combinatorial tool that we use in this paper is the classical orbit-counting lemma of Cauchy and Frobenius, which can also be found in Burnside's book [1].

Lemma 1.1. Let $\alpha$ be an action of a finite group $G$ on a finite set $A, \sim_{\alpha}$ the associated equivalence relation on $A,\left|A / \sim_{\alpha}\right|$ the number of orbits of $\alpha$, and $\operatorname{Fix}_{\alpha}(g)$ the set of elements of $A$ fixed by $g \in G$ under $\alpha$. Then

$$
\left|A / \sim_{\alpha}\right|=\frac{1}{|G|} \sum_{g \in G}\left|\operatorname{Fix}_{\alpha}(g)\right| .
$$

For a proof, see, e.g., [27, Lemma 7.24.5].

Denote by $\gamma_{\tau}(d)$ the number of non-isomorphic one-vertex oriented (pre-)maps of type $\tau$ and valence $d$, and by $\delta_{\tau}(d)$ the number of non-isomorphic one-vertex (orientable or nonorientable) (pre-)maps of type $\tau$ and valence $d$. The types of (pre-)maps that we consider are $\bar{S} \bar{D} \bar{P}, S \bar{D} \bar{P}, \bar{S} D \bar{P}, \bar{S} \bar{D} P, S D \bar{P}, S \bar{D} P, \bar{S} D P$, and $S D P$, indicating whether the underlying (pre-)graphs are signed or unsigned $(S$ resp. $\bar{S})$, directed or undirected $(D$ resp. $\bar{D})$, pre-graphs or graphs ( $P$ resp. $\bar{P})$. We write $\tau=\tau_{1} \tau_{2} \tau_{3}$ in order to be able to refer to the individual symbols which constitute the type $\tau$. As it turns out, $\gamma_{\tau}(d), \delta_{\tau}(d)$ and the various auxiliary functions can be written in the same general form for all $\tau$, but with different values of parameters (see Eqn. (2.1) for $\gamma_{\tau}(d)$, Eqns. (3.1), (2.4), (3.3), and (3.2) for $\delta_{\tau}(d)$, and Table 1 for the values of parameters).

Each oriented one-vertex pre-map is isomorphic to one of the form $(A, r, R)$ where $A=\{1,2, \ldots, d\}, R=(1,2, \ldots, d)$. Such a pre-map can be represented by a matching in the complete graph $K_{d}$ (possibly an empty one) in which two vertices $i, j \in\{1,2, \ldots, d\}$ are matched whenever $r(i)=j$. Hence the number of all one-vertex pre-maps $(A, r, R)$ with a given rotation $R$ is the same as the number of all matchings (including the empty one) in $K_{d}$. This number is easily computed to be

$$
i(d)=\sum_{0 \leq 2 k \leq d}\left(\begin{array}{c}
d \\
2 k
\end{array}\right)(2 k-1) ! !
$$

Of course, many of the above pre-maps are isomorphic. To compute the number of non-isomorphic ones, let $I_{d}$ denote the set of all permutations $r$ on $A$ with $r^{2}=1$, and recall that two oriented pre-maps $M=(A, r, R)$ and $M^{\prime}=\left(A, r^{\prime}, R\right)$ are isomorphic if and only if there exists a permutation on $A$ which centralizes (= commutes with) $R$ and conjugates $r$ to $r^{\prime}$. Note that the permutations of $A$ that centralize $R$ (the centralizer of $R$ ) form the cyclic group $C_{n}$, generated by $R$. However, if orientable maps are considered as 
general maps that reside on orientable surfaces, then the maps $(A, r, R)$ and $\left(A, r, R^{-1}\right)$ are isomorphic. In this case, an automorphism of the map is any permutation of $A$ which either commutes with $R$, or maps $R$ to $R^{-1}$. Such permutations form the dihedral group $D_{d}$ of order $2 d$, therefore the number of orientable non-isomorphic one-vertex pre-maps of valence $d$ equals the number of orbits of $D_{d}$ in its action on the set $I_{d}$ by conjugation. If the pre-maps are represented by the matchings in $K_{d}$, as described above, then the action of $D_{d}$ on $I_{d}$ corresponds to the natural action of $D_{d}$ on the set of all matchings in $K_{d}$. In general, the number $\delta_{\tau}(d)$ of non-isomorphic one-vertex pre-maps of type $\tau$ and valence $d$ equals the number of orbits of $D_{d}$ in its natural action on the set of matchings of type $\tau$ in $K_{d}$. By Lemma 1.1 we thus have:

$$
\begin{aligned}
\gamma_{\tau}(d) & =\frac{1}{\left|C_{d}\right|} \sum_{\sigma \in C_{d}}\left|\operatorname{Fix}_{\tau}(\sigma)\right|, \\
\delta_{\tau}(d) & =\frac{1}{\left|D_{d}\right|} \sum_{\sigma \in D_{d}}\left|\operatorname{Fix}_{\tau}(\sigma)\right|
\end{aligned}
$$

where $\operatorname{Fix}_{\tau}(\sigma)$ denotes the set of matchings of type $\tau$ in $K_{d}$ fixed by $\sigma$.

In all our formulæ we use the convention that $0^{0}=1$, and define the double-factorial function recursively: $n ! !=n \times(n-2)$ !! for $n \geq 1,0 ! !=(-1) ! !=1$.

\section{Cyclic symmetry: Enumeration of oriented maps and pre-maps}

Before we consider maps and pre-maps, we present simple formulæ for counting one-vertex pre-graphs and graphs.

Let $p(d)$ denote the number of one-vertex pre-graphs of valence $d$. Since each of them is determined by the number of loops, $p(d)=\lfloor d / 2\rfloor+1$. This gives rise to the generating function $P(x)=1 /\left((1-x)^{2}(1+x)\right)=1 /\left(\left(1-x^{2}\right)(1-x)\right)$.

If there are no pending edges, the situation becomes much simpler. Let $g(d)$ denote the number of one-vertex graphs. Then $g(d)=0$, for $d$ odd, and $g(d)=1$, for $d$ even. The corresponding generating function is $G(x)=1 /\left(1-x^{2}\right)$.

When extending our enumeration exercise we note immediately that the number of onevertex digraphs is the same as the number of one-vertex graphs. Also, the number of onevertex directed pre-graphs is the same as the number of one-vertex pre-graphs. Similarly, the number of one-vertex signed digraphs is the same as the number of one-vertex signed graphs, and the number of one-vertex signed directed pre-graphs is the same as the number of one-vertex signed pre-graphs. It is not hard to verify that the number of one-vertex signed graphs of valence $d$ is 0 if $d$ is odd and $d / 2+1$ if $d$ is even. The corresponding generating function is given by $1 /\left(1-x^{2}\right)^{2}$. The number of one-vertex signed pre-graphs is given by $1+2+\cdots+(\lfloor d / 2\rfloor+1)=(\lfloor d / 2\rfloor+1)(\lfloor d / 2\rfloor+2) / 2$ with the corresponding generating function $1 /\left((1-x)^{3}(1+x)^{2}\right)=1 /\left(\left(1-x^{2}\right)^{2}(1-x)\right)$.

Theorem 2.1. The number $\gamma_{\tau}(d)$ of oriented non-isomorphic one-vertex pre-maps resp. maps of type $\tau$ and valence $d$ is

$$
\gamma_{\tau}(d)=\frac{1}{d} \sum_{r \mid d} \varphi\left(\frac{d}{r}\right) \sum_{0 \leq 2 j \leq r}\left(\begin{array}{c}
r \\
2 j
\end{array}\right)(2 j-1) ! !\left(\frac{t_{\tau} d}{r}\right)^{j} w_{\tau}(r, d)^{r-2 j}
$$

where $t_{\tau}$ is given in Table 1 and $w_{\tau}(r, d)$ is shown in (2.3) below. 
Proof. As argued in the Introduction, $\gamma_{\tau}(d)$ equals the number of orbits of the cyclic group $C_{d}$ in its natural action on the set of matchings of type $\tau$ in the complete graph $K_{d}$, represented as a regular $d$-gon with diagonals. By (1.1),

$$
\gamma_{\tau}(d)=\frac{R_{\tau}(d)}{d}
$$

where $R_{\tau}(d)$ is the sum over all $\sigma \in C_{d}$ of the numbers of matchings of type $\tau$ in $K_{d}$ which are fixed by $\sigma$. To compute this number, assume that $\sigma \in C_{d}$ is the counter-clockwise rotation by $2 \pi k_{\sigma} / d$ where $k_{\sigma} \in \mathbb{Z}$ is such that $0 \leq k_{\sigma}<d$. In how many ways can one construct a matching $M$ of $K_{d}$ which is fixed by $\sigma$ ?

Let $r=\operatorname{gcd}\left(d, k_{\sigma}\right)$. Then $\sigma$ has $r$ orbits in $V\left(K_{d}\right)$, each containing $d / r$ vertices. Let $C$ denote a set of $r$ consecutive vertices of $K_{d}$. Since $C$ contains one representative from each orbit, it suffices to define $M$ on $C$, and to extend it to $V\left(K_{d}\right) \backslash C$ by symmetry. Hence we can also think of $M$ as a matching of orbits. Assume that $2 j$ of the $r$ orbits are matched in pairs, while the rest remain unmatched or are matched with themselves (the latter is possible only if antipodal vertices belong to the same orbit, i.e., if $d$ is even and $r$ divides $d / 2)$. There are $\left(\begin{array}{c}r \\ 2 j\end{array}\right)$ ways to select the $2 j$ orbits, and $(2 j-1)$ !! ways to group them into pairs. In each of the $j$ pairs of orbits $\left(\alpha_{i}, \beta_{i}\right), i=1,2, \ldots, j$, the vertex in $\alpha_{i} \cap C$ can be matched with any of the $d / r$ vertices in $\beta_{i}$ in $t_{\tau}$ ways, where the value of $t_{\tau}$ depends on the type $\tau$ of the problem considered, and is shown in Table 1. Each of the remaining $r-2 j$

\begin{tabular}{l|cccccccc}
$\tau$ & $\bar{S} \bar{D} \bar{P}$ & $S \bar{D} \bar{P}$ & $\bar{S} D \bar{P}$ & $S D \bar{P}$ & $\bar{S} \bar{D} P$ & $S \bar{D} P$ & $\bar{S} D P$ & $S D P$ \\
\hline$s_{\tau}$ & 1 & 2 & 0 & 0 & 2 & 3 & 1 & 1 \\
$t_{\tau}$ & 1 & 2 & 2 & 4 & 1 & 2 & 2 & 4 \\
$m_{\tau}$ & 1 & 2 & 2 & 4 & 2 & 3 & 3 & 5
\end{tabular}

Table 1: The values of $s_{\tau}, t_{\tau}, m_{\tau}$ for the types of (pre-)maps considered

orbits can be matched to themselves (or be left unmatched) in $w_{\tau}(r, d)$ ways where

$$
w_{\tau}(r, d)= \begin{cases}s_{\tau}, & r \mid(d / 2), \\ 0, & r \not \chi(d / 2) \text { and } \tau_{3}=\bar{P} \\ 1, & r \not \chi(d / 2) \text { and } \tau_{3}=P\end{cases}
$$

and the value of $s_{\tau}$ is given in Table 1 . For each divisor $r$ of $d$, there are $\varphi(d / r)$ rotations $\sigma$ in $D_{d}$ having $\operatorname{gcd}\left(d, k_{\sigma}\right)=r$ where $\varphi$ denotes Euler's totient function. Hence the total contribution $R_{\tau}(d)$ of the $d$ rotations is

$$
R_{\tau}(d)=\sum_{r \mid d} \varphi\left(\frac{d}{r}\right) \sum_{0 \leq 2 j \leq r}\left(\begin{array}{c}
r \\
2 j
\end{array}\right)(2 j-1) ! !\left(\frac{t_{\tau} d}{r}\right)^{j} w_{\tau}(r, d)^{r-2 j}
$$

and so by (2.2), we find that $\gamma_{\tau}(d)$ is as given in (2.1).

In Tables 2 and 3 we list the numbers $\gamma_{\tau}(d)$ for small values of $d$. 


\begin{tabular}{r|rrrr}
$d$ & $\gamma_{\bar{S} \bar{D} P}(d)$ & $\gamma_{S \bar{D} P}(d)$ & $\gamma_{\bar{S} D P}(d)$ & $\gamma_{S D P}(d)$ \\
\hline 1 & 1 & 1 & 1 & 1 \\
2 & 2 & 3 & 2 & 3 \\
3 & 2 & 3 & 3 & 5 \\
4 & 5 & 11 & 8 & 21 \\
5 & 6 & 17 & 17 & 57 \\
6 & 18 & 69 & 60 & 299 \\
7 & 34 & 187 & 187 & 1213 \\
8 & 108 & 791 & 754 & 7189 \\
9 & 294 & 2981 & 2981 & 36537 \\
10 & 984 & 13575 & 13398 & 239027 \\
11 & 3246 & 60851 & 60851 & 1412661 \\
12 & 11810 & 301937 & 300982 & 10056109 \\
13 & 43732 & 1513393 & 1513393 & 66657385 \\
14 & 171218 & 8115273 & 8109584 & 510071643 \\
15 & 689996 & 44293443 & 44293443 & 3711168173 \\
16 & 2889970 & 254424929 & 254389122 & 30257794301 \\
17 & 12458784 & 1490057537 & 1490057537 & 238049716833 \\
18 & 55415816 & 9095634067 & 9095390802 & 2053738059275 \\
19 & 253142182 & 56612205667 & 56612205667 & 17281059677029 \\
20 & 1187979372 & 364845156207 & 364843436636 & 156892675044645
\end{tabular}

Table 2: The numbers of oriented non-isomorphic one-vertex pre-maps

\begin{tabular}{r|rrrr}
$d$ & $\gamma_{\bar{S} \bar{D} \bar{P}}(d)$ & $\gamma_{S \bar{D} \bar{P}}(d)$ & $\gamma_{\bar{S} D \bar{P}}(d)$ & $\gamma_{S D \bar{P}}(d)$ \\
\hline 2 & 1 & 2 & 1 & 2 \\
4 & 2 & 6 & 4 & 14 \\
6 & 5 & 28 & 22 & 164 \\
8 & 18 & 234 & 218 & 3388 \\
10 & 105 & 3112 & 3028 & 96776 \\
12 & 902 & 55876 & 55540 & 3548876 \\
14 & 9749 & 1237648 & 1235526 & 158146572 \\
16 & 127072 & 32444680 & 32434108 & 8302721384 \\
18 & 1915951 & 980254100 & 980179566 & 501851753292 \\
20 & 32743182 & 33522615160 & 33522177088 & 34326661275896 \\
22 & 624999093 & 1279939143784 & 1279935820810 & 2621308560998420 \\
24 & 13176573910 & 53970651042864 & 53970628896500 & 221063688757926232
\end{tabular}

Table 3: The numbers of oriented non-isomorphic one-vertex maps 


\section{Dihedral symmetry: Enumeration of (non-)orientable maps and pre-maps}

Theorem 3.1. The number $\delta_{\tau}(d)$ of non-isomorphic one-vertex pre-maps resp. maps of type $\tau$ and valence $d$ is

$$
\delta_{\tau}(d)=\frac{R_{\tau}(d)+F_{\tau}(d)}{2 d}
$$

where $R_{\tau}(d)$ is given in (2.4) above, and $F_{\tau}(d)$ is given in (3.3) below.

Proof. As argued in the Introduction, $\delta_{\tau}(d)$ equals the number of orbits of the dihedral group $D_{d}$ in its natural action on the set of matchings of type $\tau$ in the complete graph $K_{d}$, represented as a regular $d$-gon with diagonals. By (1.2), this is given by the right hand-side of (3.1) where $R_{\tau}(d)$ is the sum over all rotations $\sigma \in D_{d}$, and $F_{\tau}(d)$ the sum over all reflections $\sigma \in D_{d}$, of the numbers of matchings of type $\tau$ in $K_{d}$ fixed by $\sigma$. We have already computed $R_{\tau}(d)$ in (2.4), hence it only remains to compute $F_{\tau}(d)$. We distinguish two cases according to the parity of $d$.

If $d$ is even there are two types of reflections of the regular $d$-gon: either across a median or across a main diagonal. Let $\sigma$ be the reflection across a median, and let $L$ denote the set of $n:=d / 2$ vertices of $K_{d}$ on one side of the median. Denote $f_{\tau}(n):=\left|\operatorname{Fix}_{\tau}(\sigma)\right|$. For each $u \in L$, denote by $u^{\prime}$ its mirror image across the median. In a matching $M$ which is fixed by $\sigma, u$ can be either

- left unmatched, or

- matched with $u^{\prime}$, or

- matched with some $v \in L \backslash\{u\}$, provided that $u^{\prime}$ is matched with $v^{\prime}$, or

- matched with $v^{\prime}$ for some $v \in L \backslash\{u\}$, provided that $u^{\prime}$ is matched with $v$.

Denote by $s_{\tau}$ the number of ways in which $u$ can be matched with $u^{\prime}$ (including leaving it unmatched), and by $t_{\tau}$ the number of ways in which $u$ can be matched with $v \in L \backslash\{u\}$. Because of symmetry, the number of ways in which $u$ can be matched with $v^{\prime}$ where $v \in$ $L \backslash\{u\}$ is also $t_{\tau}$. The values of $s_{\tau}$ and $t_{\tau}$ depend on the type $\tau$ of the problem considered, and are shown in Table 1.

Thus to construct all possible such matchings, select $2 j$ vertices from among the $n$ vertices in $L$, then construct a perfect matching on these $2 j$ vertices. This can be done in $\left(\begin{array}{c}n \\ 2 j\end{array}\right)(2 j-1)$ !! ways. There are $2 t_{\tau}$ ways to match the two elements in each of the $j$ pairs, yielding a factor of $\left(2 t_{\tau}\right)^{j}$, and $s_{\tau}$ ways to match each of the remaining $n-2 j$ vertices to its mirror image, yielding a factor of $s_{\tau}^{n-2 j}$. Hence

$$
f_{\tau}(n)=\sum_{0 \leq 2 j \leq n} s_{\tau}^{n-2 j}\left(2 t_{\tau}\right)^{j}\left(\begin{array}{c}
n \\
2 j
\end{array}\right)(2 j-1) ! !=n ! \sum_{0 \leq 2 j \leq n} \frac{s_{\tau}^{n-2 j} t_{\tau}^{j}}{(n-2 j) ! j !} .
$$

If $\sigma$ is the reflection across a main diagonal, then $\left|\operatorname{Fix}_{\tau}(\sigma)\right|=m_{\tau} f_{\tau}(d / 2-1)$ where $m_{\tau}$ is the number of ways in which it is possible to match the two vertices on the mirror with each other. The value of $m_{\tau}$ is shown in Table 1.

If $d$ is odd there is only one type of reflections, namely across the bisector of a side of the $d$-gon, and $\left|\operatorname{Fix}_{\tau}(\sigma)\right|=f_{\tau}((d-1) / 2)$ for pre-maps and 0 for maps. So the total 
contribution $F_{\tau}(d)$ of the $d$ reflections to the sum in (1.2) is

$$
F_{\tau}(d)= \begin{cases}\frac{d}{2}\left(f_{\tau}\left(\frac{d}{2}\right)+m_{\tau} f_{\tau}\left(\frac{d}{2}-1\right)\right), & d \text { even, } \\ d f_{\tau}\left(\frac{d-1}{2}\right), & d \text { odd and } \tau_{3}=P, \\ 0, & d \text { odd and } \tau_{3}=\bar{P}\end{cases}
$$

where $f_{\tau}$ is given by (3.2), and $m_{\tau}$ is shown in Table 1 . This finishes the proof of the theorem.

In Tables 4 and 5 we list the numbers $\delta_{\tau}(d)$ for small values of $d$. For instance, $\delta_{\bar{S} \bar{D} P}(5)=6$, so there are six pairwise non-isomorphic one-vertex undirected orientable pre-maps of valence five (which, incidentally, are discussed in detail in [24]).

\section{Concluding remarks}

\subsection{Additional formulæ}

In the special case when $w_{\tau}(r, d)=0$ for all $r$, the double sum in (2.4) reduces to a single sum. For $d$ even we thus have

$$
\begin{aligned}
R_{\bar{S} D \bar{P}}(d) & =\sum_{r \mid d, r \text { even }} \varphi\left(\frac{d}{r}\right)(r-1) ! !\left(\frac{2 d}{r}\right)^{r / 2} \\
R_{S D \bar{P}}(d) & =\sum_{r \mid d, r \text { even }} \varphi\left(\frac{d}{r}\right)(r-1) ! !\left(\frac{4 d}{r}\right)^{r / 2}
\end{aligned}
$$

It is not difficult to see that the sequence $f_{\tau}(n)$ from (3.2) satisfies the recurrence

$$
f_{\tau}(n)=s_{\tau} f_{\tau}(n-1)+2 t_{\tau}(n-1) f_{\tau}(n-2), \quad \text { for } n \geq 2,
$$

with $f_{\tau}(0)=1, f_{\tau}(1)=s_{\tau}$, and that its exponential generating function equals

$$
\sum_{n=0}^{\infty} f_{\tau}(n) \frac{x^{n}}{n !}=\exp \left(s_{\tau} x+t_{\tau} x^{2}\right) .
$$

Comparing this with the well-known exponential generating function of Hermite polynomials

$$
\sum_{n=0}^{\infty} H_{n}(t) \frac{x^{n}}{n !}=\exp \left(2 t x-x^{2}\right),
$$

we see that

$$
f_{\tau}(n)=\left(i \sqrt{t_{\tau}}\right)^{n} H_{n}\left(\frac{s_{\tau}}{2 i \sqrt{t_{\tau}}}\right)
$$

where $i^{2}=-1$ and $H_{n}(t)$ is the $n$-th Hermite polynomial. In the special case when $s_{\tau}=0$, the sum in (3.2) has a single term. Thus, for $n$ even,

$$
\begin{aligned}
& f_{\bar{S} D \bar{P}}(n)=2^{n}(n-1) ! ! \\
& f_{S D \bar{P}}(n)=(2 \sqrt{2})^{n}(n-1) ! !
\end{aligned}
$$




\begin{tabular}{r|rrrr}
$d$ & $\delta_{\bar{S} \bar{D} P}(d)$ & $\delta_{S \bar{D} P}(d)$ & $\delta_{\bar{S} D P}(d)$ & $\delta_{S D P}(d)$ \\
\hline 1 & 1 & 1 & 1 & 1 \\
2 & 2 & 3 & 2 & 3 \\
3 & 2 & 3 & 2 & 3 \\
4 & 5 & 11 & 6 & 14 \\
5 & 6 & 15 & 11 & 33 \\
6 & 17 & 60 & 37 & 167 \\
7 & 27 & 125 & 100 & 619 \\
8 & 83 & 529 & 1505 & 3686 \\
9 & 185 & 1663 & 6824 & 18389 \\
10 & 608 & 7557 & 30566 & 706851 \\
11 & 1779 & 31447 & 151137 & 5032026 \\
12 & 6407 & 155758 & 757567 & 33334033 \\
13 & 22558 & 763211 & 4058219 & 255064335 \\
14 & 87929 & 4089438 & 22150964 & 1855614411 \\
15 & 348254 & 22190781 & 127215233 & 15129137658 \\
16 & 1456341 & 127435846 & 745057385 & 119025187809 \\
17 & 6245592 & 745343353 & 4547820514 & 1026870988199 \\
18 & 27766356 & 4549465739 & 28306267210 & 8640532108675 \\
19 & 126655587 & 28308456491 & 182422562168 & 78446356190934 \\
20 & 594304478 & 182435301597 & &
\end{tabular}

Table 4: The numbers of non-isomorphic one-vertex pre-maps

\begin{tabular}{r|rrrr}
$d$ & $\delta_{\bar{S} \bar{D} \bar{P}}(d)$ & $\delta_{S \bar{D} \bar{P}}(d)$ & $\delta_{\bar{S} D \bar{P}}(d)$ & $\delta_{S D \bar{P}}(d)$ \\
\hline 2 & 1 & 2 & 1 & 2 \\
4 & 2 & 6 & 3 & 9 \\
6 & 5 & 26 & 13 & 90 \\
8 & 17 & 173 & 121 & 1742 \\
10 & 79 & 1844 & 1538 & 48580 \\
12 & 554 & 29570 & 28010 & 1776358 \\
14 & 5283 & 628680 & 618243 & 79080966 \\
16 & 65346 & 16286084 & 16223774 & 4151468212 \\
18 & 966156 & 490560202 & 490103223 & 250926306726 \\
20 & 16411700 & 16764409276 & 16761330464 & 17163338379388 \\
22 & 312700297 & 639992710196 & 639968394245 & 1310654311464970 \\
24 & 6589356711 & 26985505589784 & 26985325092730 & 110531845060209836
\end{tabular}

Table 5: The numbers of non-isomorphic one-vertex maps 


\subsection{Identification of sequences in OEIS}

Some of the sequences encountered in this paper can be found in the The Online Encyclopedia of Integer Sequences [25], to wit:

- $\left\langle\delta_{\bar{S} \bar{D} \bar{P}}(2 n)\right\rangle_{n=1}^{\infty}$ is sequence A054499 in [25],

- $\left\langle\gamma_{\bar{S} \bar{D} \bar{P}}(2 n)\right\rangle_{n=1}^{\infty}$ is sequence A007769 in [25],

- $\left\langle f_{\bar{S} \bar{D} \bar{P}}(n)\right\rangle_{n=0}^{\infty}$ is sequence A047974 in [25],

- $\left\langle f_{\bar{S} \bar{D} P}(n)\right\rangle_{n=0}^{\infty}$ is sequence A000898 in [25],

- $\left\langle f_{\bar{S} D P}(n)\right\rangle_{n=0}^{\infty}$ is sequence A115329 in [25],

- $\left\langle f_{\bar{S} D \bar{P}}(2 n-2)\right\rangle_{n=1}^{\infty}$ is sequence A052714 in [25],

- $\left\langle f_{S D \bar{P}}(2 n-2)\right\rangle_{n=1}^{\infty}$ is sequence A052734 in [25].

\subsection{Possible extensions}

Using the methods of [18] one can easily extend the counting to graphs with one-vertex connected components. For a more recent example of such methods, see also [16]. Motivated by [14], it would be worthwhile to extend this analysis to dipoles or any two-vertex graphs or pre-graphs.

\section{Acknowledgements}

The authors would like to thank T.W. Tucker for his helpful remarks and to the anonymous referees for their valuable suggestions which helped improve the quality of the paper.

\section{References}

[1] W. Burnside, Theory of Groups of Finite Order, second ed. (1911), Cambridge University Press, 1897.

[2] G. Chapuy, The structure of unicellular maps, and a connection between maps of positive genus and planar labelled trees, arXiv:0804.0546v2 [math.CO].

[3] R. Cori, Un code pour les graphes planaires et ses applications, Astérisque, No. 27, Société Mathématique de France, Paris, 1975.

[4] R. Cori and M. Marcus, Counting non-isomorphic chord diagrams, Theoret. Comput. Sci. 204 (1998), 55-73.

[5] C. D. Godsil and G. Royle, Algebraic Graph Theory, Springer Verlag, New York, Berlin, Heidelberg, 2001.

[6] I. P. Goulden and A. Nica, A direct bijection for Harer-Zagier formula, J. Combin. Theory Ser. A 111 (2005), 224-238.

[7] J. Gross, D. P. Robbins and T. W. Tucker, Genus distributions for bouquets of circles, J. Combin. Theory Ser. B 47 (1989), 292-306.

[8] J. Gross and T. W. Tucker, Topological Graph Theory, Wiley Interscience, 1987.

[9] F. Harary and E. M. Palmer, Graphical Enumeration, Academic Press, 1973.

[10] J. Harer and D. Zagier, The Euler characteristic of the moduli space of curves, Invent. Math. 85 (1986), 457-486.

[11] L. Heffter, Über das Problem der Nachbargebiete, Math. Ann. 38 (1891), 477-508. 
[12] A. Jacques, Sur le genre d'une paire de substitutions, C. R. Acad. Sci. Paris 267 (1968), 625627.

[13] A. Khruzin, Enumeration of chord diagrams, arXiv:math/0008209v1 [math.CO].

[14] J. H. Kwak and S. H. Shim, Total embedding distributions for bouquets of circles, Discrete Math. 248 (2002), 93-108.

[15] V. A. Liskovets, Reductive enumeration under mutually orthogonal group actions, Acta Appl. Math. 52 (1998), 91-120.

[16] V. A. Liskovets and A. D. Mednykh, On the number of connected and disconnected coverings over a manifold, Ars Math. Contemp. 2 (2009), 181-189.

[17] A. Malnič, R. Nedela and M. Škoviera, Regular homomorphisms and regular maps, Europ. J. Combin. 23 (2002), 449-461.

[18] M. Petkovšek and T. Pisanski, Counting disconnected structures: chemical trees, fullerenes, I-graphs, and others, Croat. Chem. Acta 78 (2005), 563-567.

[19] T. Pisanski, A classification of cubic bicirculants, Discrete Math. 307 (2007), 567-578.

[20] T. Pisanski and P. Potočnik, Graphs on surfaces, in: J.L. Gross, J. Yellen (eds.), Handbook of Graph Theory, The CRC Press Series on Discrete Mathematics and its Applications, CRC Press, Boca Raton, 2004, 611-624.

[21] R. B. Richter, J. Širáň, R. Jajcay, T. W. Tucker and M. E. Watkins, Cayley maps, J. Combin. Theory Ser. B 95 (2005), 189-245.

[22] G. Ringel, Map Color Theorem, Die Grundlehren der mathematischen Wissenschaften, Band 209, Springer-Verlag, New York-Heidelberg, 1974.

[23] R. W. Robinson, Counting perfect matchings with superposed directed cycles, presented at the 21st Cumberland Conference on Graph Theory, Combinatorics, and Computing - In Honor of Mike Plummer's 70th Birthday May 15-17, 2008, Vanderbilt University, Nashville, TN, USA

[24] J. Šiagiová and Mark E. Watkins, Covalence sequences of planar vertex-homogeneous maps, Discrete Math. 307 (2007), 599-614.

[25] N. J. A. Sloane, The On-Line Encyclopedia of Integer Sequences, published electronically at http://www.research.att.com/ njas/sequences/.

[26] S. Stahl, Generalized embedding schemes, J. Graph Theory 2 (1978), 41-52.

[27] R. P. Stanley, Enumerative Combinatorics, vol. 2, Cambridge University Press, Cambridge 1999.

[28] A. Stoimenow, On the number of chord diagrams, Discrete Math. 218 (2000), 109-233.

[29] W. T. Tutte, What is a map?, New Directions in the Theory of Graphs (Proc. Third Ann Arbor Conf., Univ. Michigan, Ann Arbor, Mich., 1971), pp. 309-325. Academic Press, New York, 1973.

[30] T. R. S. Walsh and A. B. Lehman, Counting rooted maps by genus. I, J. Combin. Theory Ser. B 13 (1972), 192-218.

[31] A. T. White, Graphs of Groups on Surfaces, North-Holland Mathematics Studies 188, NorthHolland Publishing Co., Amsterdam 2001. 